\title{
Contributing Authors
}

\section{Drusilla K. Brown}

Department of Economics-A\&S

Tufts University

Braker Hall

Medford, MA 02155

dbrown04@emerald.tufts.edu

\section{Wendy Dobson}

University of Toronto

105 St. George Street

Toronto, Ontario MSS 3E6

CANADA

dobson@epas.utoronto.ca

\section{Bruce Gardner}

University of Maryland

2105 Sumons Hall

College Park, MD 20742

bruce@arec.umd.edu

\section{James E. Harrigan}

International Research Department

Federal Reserve Bank of New York

33 Liberty Street, 3rd Floor

New York, NY 10045-1003

james.harrigan@ny.frb.org

\section{Thomas W. Hertel}

Center For Global Trade Analysis

Purdue University

1145 Krannert Building

West Lafayette, IN 47907-1145

hertel@agecon.purdue.edu

\section{Bernard Hoekman}

International Trade Group

The World Bank

1818 H St. NW

Washington, DC 20423

bhoekman@worldbank.org

\section{Timothy Josling}

Stanford University

Encina Hall, Room E113

616 Serra Street

Stanford, CA 94305

josling@leland.stanford.edu
Keith E. Maskus

Department of Economics

University of Colorado

Box 256

Boulder, CO 80309

kentl.maskus@colorado.edu

\section{Geoffrey Reed}

School of Economics

University of Nottingham

Nottingham, NG7 2RD

ENGLAND

geoffrey.reed@nottingham.ac.uk

\section{J. David Richardson}

Economics, Maxwell School

Syracuse University

303 Browne Hall

Syracuse, NY 13244

jdrichar@maxwell.syr.edu

\section{Jeffrey J. Schott}

Institute of International Economics

11 Dupont Circle, NW, Suite 620

Washington, DC 20036-1207

jschott@iie.com

\section{T. N. Srinivasan}

Economic Growth Center

Yale University

27 Hillhouse Avenue

New Haven, CT 06520-8269

t.srinivasan@yale.edu 
REVIEW 